\title{
EFEITOS DO SUPERAQUECIMENTO NOS PARÂMETROS TÉRMICOS E SUA CORRELAÇÃO COM A MACRO, MICROESTRUTURA E MICRODUREZA HV DA LIGA AI-3\%Mg SOLIDIFICADA UNIDIRECIONALMENTE*
}

\author{
Eduardo Reis de Oliveira ${ }^{1}$ \\ Fernando Tadeu de Araújo Filho ${ }^{2}$ \\ Estefano Aparecido Vieira ${ }^{3}$ \\ Claudio Alves de Siqueira Filho ${ }^{4}$
}

Resumo

As ligas fundidas de Alumínio são uma excelente opção para substituir os materiais convencionais devido às suas excelentes propriedades mecânicas. Assim, o uso destas ligas nas indústrias aeroespacial e automobilística estão em uso crescente. As propriedades mecânicas finais das peças são muito dependentes da macro e microestrutura, que são resultantes do processo de solidificação. Portanto, o controle dos processos de solidificação de ligas permite maximizar as propriedades mecânicas de um componente mecânico. Os parâmetros de processo que podem interferir na estrutura final de uma liga são amplos. Tem-se como exemplo: composição da liga, taxa de resfriamento, modo de extração de calor, condições de fusão e tratamento do metal líquido e temperaturas envolvidas durante a fundição. O objetivo deste trabalho foi estudar uma liga binária de $\mathrm{Al}-\mathrm{Mg}$ usando a técnica solidificação unidirecional. As macroestruturas e microestruturas obtidas foram correlacionadas com parâmetros térmicos tais como: velocidade de deslocamento da isoterma liquidus $\left(\mathrm{V}_{\mathrm{L}}\right)$ e taxa de resfriamento $(\dot{T})$. $\mathrm{E}$ os espaçamentos dendrítico secundários $\left(\lambda_{2}\right)$ com a microdureza HV. A composição da liga analisada foi $\mathrm{Al}-\mathrm{Mg} \mathrm{com} 3 \%$ de $\mathrm{Mg}$ e com patamares de superaquecimento $(\Delta \mathrm{T} v)$ variando em 20, 40 e $60^{\circ} \mathrm{C}$.

Palavras-chave: Solidificação Direcional; Macroestrutura; Microestrutura; Microdureza HV.

\section{EFFECTS OF OVERHEATING IN THERMAL PARAMETERS AND ITS CORRELATION WITH MACRO-STRUCTURE, MICROSTRUCTURE AND MICRO- HARDNESS HV OF AL-3\%MG ALLOY DIRECTLY SOLIDIFIED}

\begin{abstract}
Aluminum cast alloys are an excellent choice to replace conventional materials because of their excellent mechanical properties. Thus the use of these alloys in the aerospace and automotive industries are in increasing use. The final mechanical properties of the parts are very dependent on the macro and microstructure, which are the result of the solidification process. Therefore, the control of the solidification processes of alloys allows to maximize the mechanical properties of a mechanical component. The process parameters that can interfere in the final structure of an alloy are ample. Examples are: alloy composition, cooling rate, mode of heat extraction, melting conditions and treatment of liquid metal and temperatures involved during casting. The objective of this work was to study a binary Al-Mg alloy using the unidirectional solidification technique. The obtained macroestructures and microstructures were correlated with thermal parameters such as: velocity of displacement of liquidus isotherm $(\mathrm{VL})$ and cooling rate $(\dot{T})$. And the secondary dendritic spacings $(\lambda 2)$ with the microhardness HV. The composition of the alloy analyzed was $\mathrm{Al}-\mathrm{Mg}$ with $3 \% \mathrm{Mg}$ and with superheating levels $(\Delta \mathrm{TV})$ varying at 20,40 and $60^{\circ} \mathrm{C}$.

Keywords: Directional Solidification; Macroestrutura; Microstructure; Microhardness HV.

Eng. Metalúrgico, Mestre, Doutorando, PPGEM, Univ. Federal da Paraíba (UFPA), João Pessoa, PA, Brasil.

Graduando em Engenharia de Materiais, Graduando, DEMAT, UFPA, João Pessoa, PA, Brasil.

Eng. Metalúrgico, Doutor, Professor, PPGEMM, Instituto Federal do Espirito Santo (IFES), Vitoria, ES, Brasil.

Eng. Mecânico, Doutor, Professor, PPGEM, UFPA, João Pessoa, Paraíba, Brasil.
\end{abstract}




\section{INTRODUÇÃO}

Nas ligas de alumínio, é fato conhecido que as propriedades mecânicas, são dependentes da estrutura formada após a solidificação. A obtenção de estruturas de solidificação compatíveis com as propriedades mecânicas exigidas é o objetivo fundamental dos estudos realizados neste campo, pois sabe-se que o posterior desempenho das peças obtidas é significativamente influenciado pela estrutura bruta constituída imediatamente após a solidificação.

Em geral, no caso da macroestrutura três zonas distintas podem ser identificadas: zona coquilhada, zona colunar e zona equiaxial. As três zonas podem não estar presentes em um mesmo caso; entretanto, quando um metal fundido contém apenas grãos colunares e equiaxiais, a transição entre as duas morfologias dá-se em uma faixa estreita, com as zonas completamente distintas. A determinação deste plano, é chamado de Transição da Estrutura Colunar-Equiaxial.

Em relação a microestrutura, os espaçamentos dendríticos primários e secundários são dados importantes para as características mecânicas do material. A literatura relata que os espaçamentos microestruturais diminuem com o aumento da velocidade de deslocamento do isoterma liquidus $\left(\mathrm{V}_{\mathrm{L}}\right)$ e da Taxa de Resfriamento $(\dot{T})$ (Hunt, [1]). Desta forma, sistemas de solidificação que favoreçam tais condições, ou seja, que promovam a formação de microestruturas refinadas, contribuem para a obtenção de produtos de melhor resistência mecânica (Cruz [2] e Reis [3]).

A solidificação dos metais pode ser realizada em regime estacionário ou transitório de calor (Bouchard e Kirkaldy [4]). Os estudos utilizando sistemas de solidificação de ligas com configuração unidirecional em condições transientes de extração de calor, estão cada vez mais sendo pesquisados por diversos autores (Gafur et al [5], Cheung [6] e Siquera [7]) com objetivo de se obter macroestruturas colunares. Existem muitas investigações relacionadas com a transferência de calor sob estas condições que por sua vez mostram a importância de se desenvolver ferramentas que permitam prever a troca térmica em função das variáveis envolvidas nos processos de fundição e solidificação (Spinelli et al [8]).

Nas ligas $\mathrm{Al}-\mathrm{Mg}$, o magnésio confere um elevado nível de ductilidade assim como melhora a resistência a corrosão e soldabilidade. Assim, neste trabalho foi realizado um estudo da influência dos superaquecimentos em 20,40 e $60^{\circ} \mathrm{C}$ nos parâmetros térmicos $V_{L}$ e $\dot{T}$ e suas correlações com parâmetros estruturais e propriedades mecânica, para a liga Al-3\%Mg.

\section{MATERIAIS E MÉTODOS}

\section{2 .1 Elaboração das ligas}

Inicialmente, as ligas foram obtidas a partir da fusão do alumínio a $760^{\circ} \mathrm{C} \mathrm{com}$ a posterior adição de 3\% de Magnésio. A tabela 1 apresenta a composição química dos lingotes obtidos que basicamente são constituídos de alumínio, magnésio e impurezas dentro dos limites estabelecidos para ligas comerciais.

Tabela 1: Análise Química da composição da liga estudada ( $\mathrm{Al}$ - 3\%Mg) \% em massa.

\begin{tabular}{|c|c|c|c|c|c|c|c|c|}
\hline LIGA & Al & Fe & Cu & Mn & Mg & Zn & Si & Cr \\
\hline Al-3\%Mg & 96,7 & 0,0581 & 0,0005 & 0,0064 & 3,09 & 0,0063 & 0,0444 & 0,0024 \\
\hline
\end{tabular}

\subsection{Elaboração dos lingotes.}


Após a refusão das ligas e atingir o superaquecimento desejado, estas foram vazadas

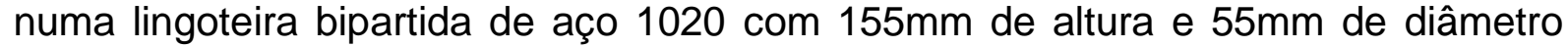
interno, posicionada dentro de um forno de Solidificação Direcional (SD) ascendente e refrigerado a água conforme mostra esquematicamente a Figura 1.0 forno então é desligado e injetado um fluxo de água na base da lingoteira. Durante o resfriamento as temperaturas dos lingotes foram monitoradas por 5 termopares do tipo $\mathrm{K}$ conectados a um registrador de temperaturas com interface on-line a um computador, onde os dados foram armazenados. Assim, foi possível determinar a velocidade da isoterma liquidus e da taxa de resfriamento. Os termopares foram posicionados a 5 , $10,35,50$ e $70 \mathrm{~mm}$ da interface metal/ molde refrigerada (base da lingoteira). As posições dos termopares durante os trabalhos experimentais foram definidas visando ao máximo obedecerem às possibilidades unidirecionais de extração de calor, bem como a importância dos instantes iniciais do processo de solidificação.

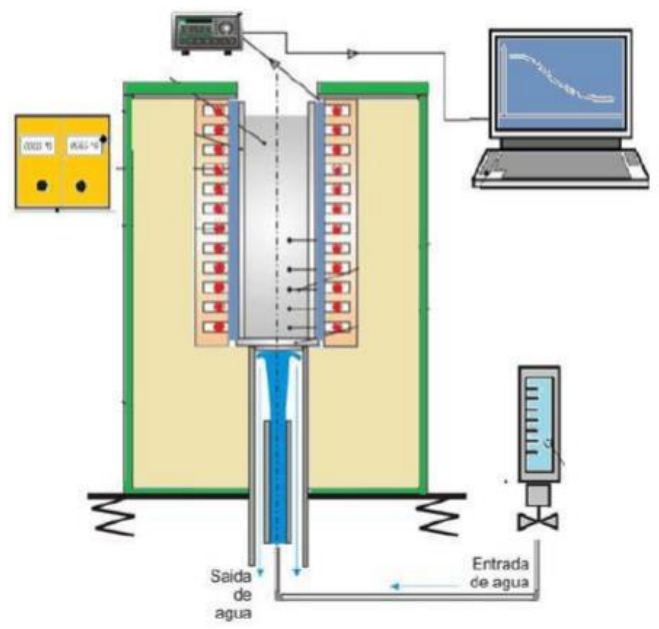

Figura 1: Dispositivo de solidificação unidirecional ascendente. Dantas [9]

\subsection{Analise macroestrutural.}

Os lingotes obtidos foram seccionados longitudinalmente, sendo uma das partes utilizada para a caracterização da macroestrutura, e a outra para confeccionar o corpo de prova para o ensaio de microdureza Vickers. Após este procedimento, as amostras foram lixadas em uma sequência crescente de lixas d'agua (200 a 600 mesh). Após serem lixadas receberam ataque químico por fricção em uma solução aquosa com a seguinte composição: $10 \mathrm{ml} \mathrm{HF}, 25 \mathrm{ml} \mathrm{HNO}_{3}, 15 \mathrm{ml} \mathrm{HCl}$ e $50 \mathrm{ml} \mathrm{H} \mathrm{H}_{2}$ (Solução de Keller) durante 30 segundos. $O$ resultado do ataque químico consistiu na revelação da macroestrutura.

\subsection{Análise microestrutural.}

Para a análise da microestrutura as amostras foram lixadas (600 a 1200 mesh), e posteriormente polidas com pasta de diamante de 1 mícron. Em seguida foram atacadas com uma solução de $10 \mathrm{ml} \mathrm{HF}, 15 \mathrm{ml} \mathrm{HNO}_{3}$, e $75 \mathrm{ml} \mathrm{H} \mathrm{H}_{2} \mathrm{O}$ (Solução de Kroll) durante 60 segundos. Após o ataque as medições dos espaçamentos dendríticos primários foram realizadas sobre as amostras transversais dos lingotes, utilizando 0 método triângulo (Gunduz e Çadirlli [10]) e os espaçamentos dendríticos secundários foram realizados sobre as amostras longitudinais dos lingotes, utilizando o método da intercepção. Foram realizadas em média, 20 medições para cada posição analisada, em seguida obtida a média dos valores e os respectivos intervalos de dispersão. 


\subsection{Ensaios de microdureza HV.}

Os ensaios de microdureza Vickers (HV) foram realizados com a aplicação de uma carga de 0,5 kgf durante 15 segundos em um microdurômetro da marca Shimadzu, modelo HMV-2T. Foram realizadas 20 leituras para cada amostra e, ao final, foram obtidos a média dos valores e o respectivo intervalo de dispersão. Este ensaio é padronizado pela norma ASTM E384.

\section{RESULTADOS E DISCUSSÃO}

\subsection{Variáveis térmicas de solidificação.}

Para cada superaquecimento da liga analisada, foram obtidos os mapeamentos de temperatura para 5 posições dos termopares a partir da interface metal/molde (base refrigerada). A partir das curvas de resfriamento foi possível estabelecer a relação posição/tempo para os três superaquecimentos estudados da liga Al-3\%Mg. A Figura 2 apresenta os resultados experimentais da curva Posição x Tempo, onde: $\mathrm{P}$ é a posição da linha liquidus no lingote a partir da base refrigerada em ( $\mathrm{mm})$, e t é o tempo em (s). Estes pontos foram obtidos a partir das interseções das retas de cada temperatura liquidus $\left(\mathrm{T}_{\mathrm{L}}\right)$ com curvas de resfriamento para cada posição dos termopares. Sendo Tv a temperatura de vazamento para cada um dos três superaquecimentos $(\Delta T \mathrm{~T})$ propostos.
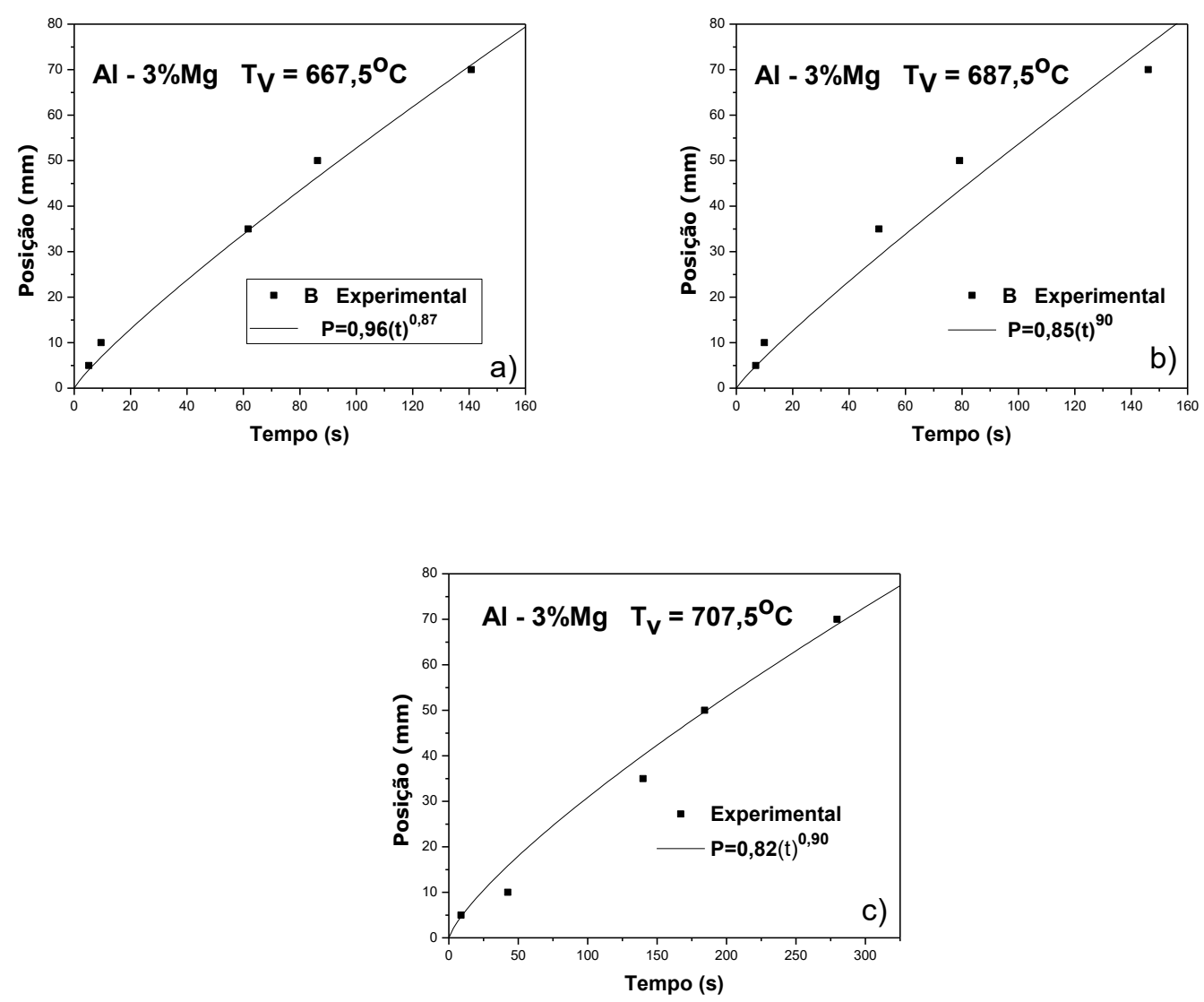

Figura 2: Posição da interface liquidus versus tempo para a liga $\mathrm{Al}-3 \% \mathrm{Mg}$ - a) $\Delta \mathrm{TV}=20^{\circ} \mathrm{C}$; b) $\Delta \mathrm{TV}=$ $40^{\circ} \mathrm{C}$; c) $\Delta \mathrm{TV}=60^{\circ} \mathrm{C}$ respectivamente.

A derivada da equação da posição com relação ao tempo, ou seja, $V_{L}=d P / d t$, permitiu a obtenção dos respectivos valores experimentais para as velocidades de deslocamento da isoterma liquidus a partir da interface metal/molde em função do 
tempo, para os superaquecimentos liga investigada, conforme mostra a Figura 3. A velocidade da isoterma liquidus para os superaquecimentos de 20,40 e $60^{\circ} \mathrm{C}$ da liga $\mathrm{Al}-3 \% \mathrm{Mg}$. Nota-se que os perfis de velocidade para ambos superaquecimentos são bem próximos. Observou-se ainda que $V_{L}$ diminui para as posições mais afastadas da base refrigerada até permanecer a um valor constante na altura $70 \mathrm{~mm}$ da base refrigerada, como ilustra o fenômeno a Figura 3. Pode-se afirmar que os espaçamentos dendríticos a partir desta altura também permanecem constantes.

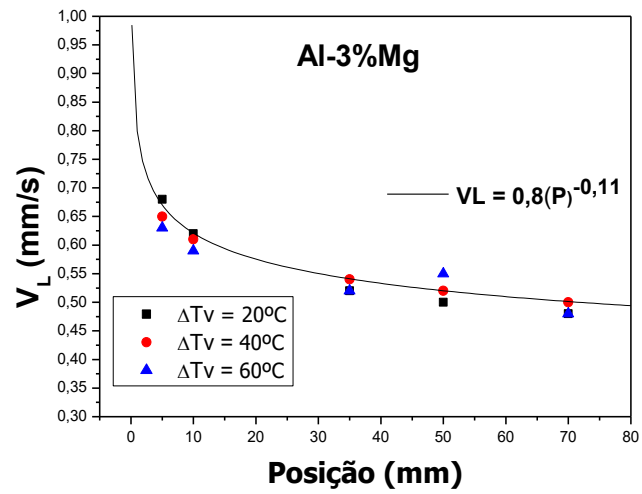

Figura 3: Velocidade da isoterma Liquidus $\left(V_{\mathrm{L}}\right) \times$ Posição da interface $S / L$ da liga com Al-3\% Mg, com os superaquecimentos $(\Delta \mathrm{T} v)=20,40$ e $60^{\circ} \mathrm{C}$.

Na figura 4 é apresentado a evolução da taxa de resfriamento à frente da interface metal/molde durante a solidificação para os três superaquecimentos estudados da liga $\mathrm{Al}-3 \% \mathrm{Mg}$.

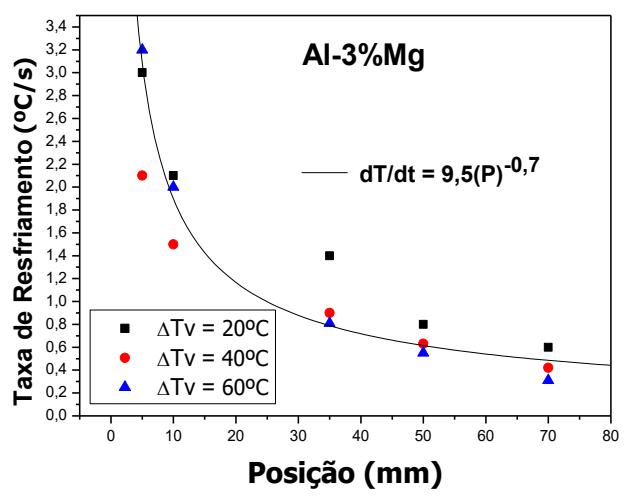

Figura 4: Curva da taxa de resfriamento $(\dot{T}) \times$ Posição da interface $S / L$ dá liga com Al- $3 \%$ de $\mathrm{Mg}$, com os superaquecimentos $(\Delta \mathrm{TV})=20,40$ e $60^{\circ} \mathrm{C}$.

\subsection{Macroestruturas de solidificação.}

A Figura 5 mostra que para todos os experimentos foi obtido uma ampla faixa de estrutura colunar. Conforme observação nos lingotes a variação do superaquecimento em 20,40 e $60^{\circ} \mathrm{C}$ respectivamente, não gerou alteração significativa na altura onde ocorreu a Transição Colunar Equiaxial (TCE). 
a)

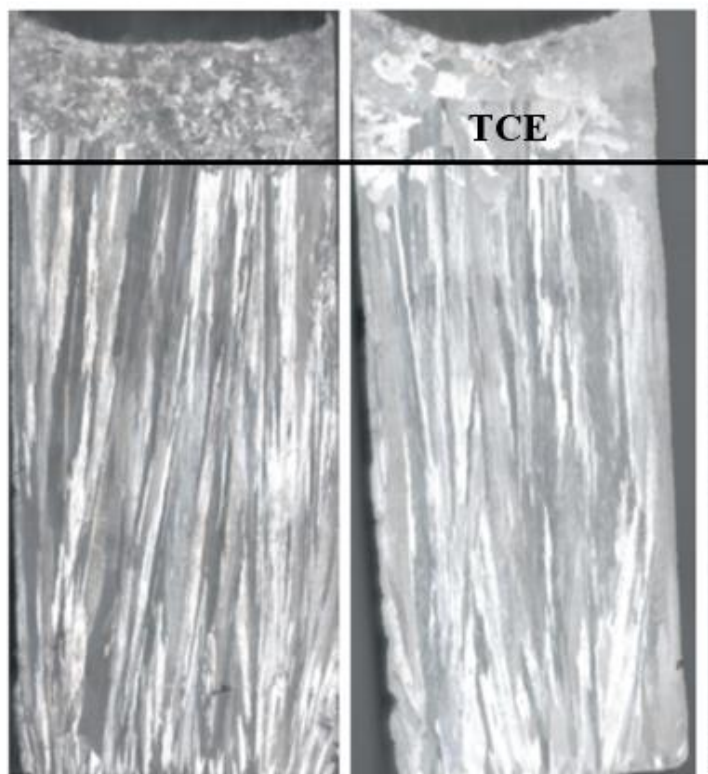

c)

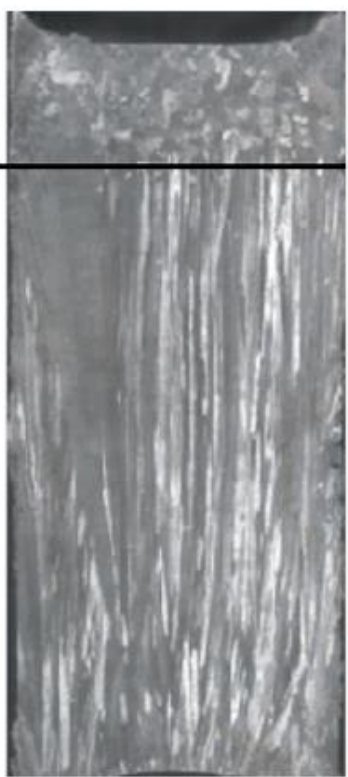

Figura 5: Macroestruturas reveladas da liga $\mathrm{Al}-3 \% \mathrm{Mg}$ : a) $\Delta \mathrm{T}_{\mathrm{v}}=20^{\circ} \mathrm{C}$; b) $\Delta \mathrm{T} v=40^{\circ} \mathrm{C}$; c) $\Delta \mathrm{T} v=60^{\circ} \mathrm{C}$.

Na tabela 2 são apresentados os parâmetros térmicos onde ocorreu a Transição Colunar-Equiaxial para os três patamares de superaquecimentos estudados.

Tabela 2: Parâmetros térmicos e altura da TCE em função dos superaquecimentos da liga $\mathrm{Al}-3 \% \mathrm{Mg}$ investigados.

\begin{tabular}{|c|c|c|c|}
\hline$\Delta$ Tv $\left({ }^{\circ} \mathbf{C} / \mathbf{s}\right)$ & $\begin{array}{c}\text { Posição } \\
\text { TCE }(\mathbf{m m})\end{array}$ & $\begin{array}{c}\text { Velocidade Isoterma } \\
\text { Liquidus } \mathbf{V}_{\mathbf{L}}(\mathbf{m m} / \mathbf{s})\end{array}$ & $\begin{array}{c}\text { Taxa de } \\
\text { Resfriamento } \\
\dot{\boldsymbol{T}}(\mathbf{~} \mathbf{C} / \mathbf{s})\end{array}$ \\
\hline $\mathbf{2 0}$ & 125 & 0,48 & 0,26 \\
\hline $\mathbf{4 0}$ & 125 & 0,47 & 0,24 \\
\hline $\mathbf{6 0}$ & 125 & 0,47 & 0,23 \\
\hline
\end{tabular}

Para todos os três superaquecimentos a posição da TCE ocorreu a $125 \mathrm{~mm}$ da base refrigerada dos lingotes, nos seguintes parâmetros térmicos: $\Delta T_{V}=20^{\circ} \mathrm{C}\left(V_{L}=\right.$ $0,48 \mathrm{~mm} / \mathrm{s}$ e $\dot{T}=0,26), \Delta \mathrm{T}_{\mathrm{V}}=40^{\circ} \mathrm{C}\left(\mathrm{V}_{\mathrm{L}}=0,47 \mathrm{~mm} / \mathrm{s}\right.$ e $\left.\dot{T}=0,24\right)$ e para $\Delta \mathrm{TV}_{\mathrm{V}}=60^{\circ} \mathrm{C}\left(\mathrm{V}_{\mathrm{L}}\right.$ $=0,47 \mathrm{~mm} / \mathrm{s}$ e $\dot{T}=0,23$ ) respectivamente.

\subsection{Microestruturas de solidificação.}

Estão apresentados na Figura 6 as microestruturas reveladas das posições 5 e $70 \mathrm{~mm}$ em relação a base refrigerada dos lingotes estudados. São representadas para cada posição os (EDP ou $\lambda_{1}$ ) nas seções transversais, a esquerda e os (EDS ou $\lambda_{2}$ ) nas seções longitudinais, a direita. 

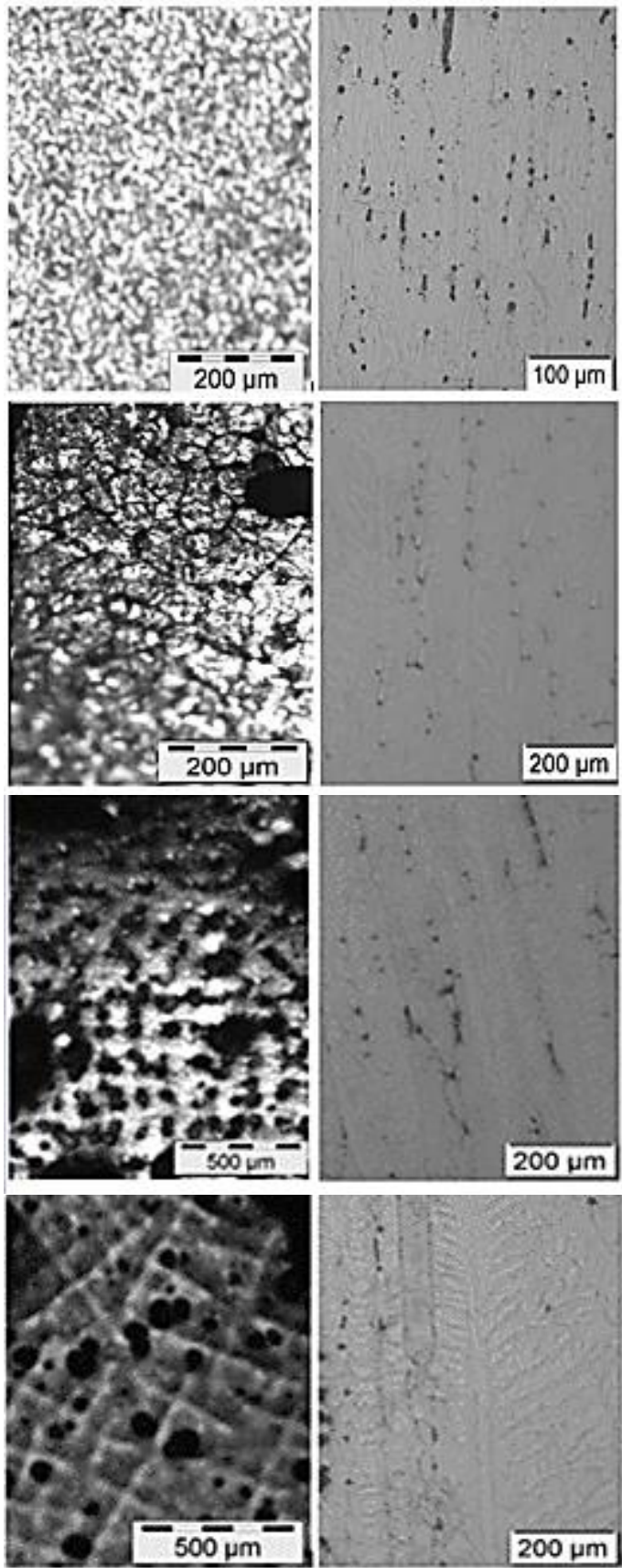

$$
\begin{aligned}
& \mathrm{P}=5 \mathrm{~mm} ; \\
& \dot{T}=2,81^{\circ} \mathrm{c} / \mathrm{s} ; \\
& \mathrm{V}_{\mathrm{L}}=0,48 \mathrm{~mm} / \mathrm{s} ; \\
& \lambda_{1}=60,35 \mu \mathrm{m} ; \\
& \lambda_{2}=26,01 \mu \mathrm{m} .
\end{aligned}
$$

a)

$$
\begin{aligned}
& \mathrm{P}=70 \mathrm{~mm} ; \\
& \dot{T}=0,51^{\circ} \mathrm{C} / \mathrm{s} ; \\
& \mathrm{V}_{\mathrm{L}}=0,25 \mathrm{~mm} / \mathrm{s} ; \\
& \lambda_{1}=258,22 \mu \mathrm{m} ; \\
& \lambda_{2}=41,91 \mu \mathrm{m} .
\end{aligned}
$$

b)

$$
\begin{aligned}
& \mathrm{P}=5 \mathrm{~mm} ; \\
& \dot{T}=2,81^{\circ} \mathrm{C} / \mathrm{s} ; \\
& \mathrm{V}_{\mathrm{L}}=0,44 \mathrm{~mm} / \mathrm{s} ; \\
& \lambda_{1}=65,51 \mu \mathrm{m} ; \\
& \lambda_{2}=27,4 \mu \mathrm{m} .
\end{aligned}
$$

c)

$$
\begin{aligned}
& \mathrm{P}=70 \mathrm{~mm} ; \\
& \dot{I}=0,50^{\circ} \mathrm{C} / \mathrm{s} ; \\
& \mathrm{V}_{\mathrm{L}}=0,30 \mathrm{~mm} / \mathrm{s} ; \\
& \lambda:=267,10 \mu \mathrm{m} ; \\
& \lambda_{2}=44,41 \mu \mathrm{m} .
\end{aligned}
$$

d) 

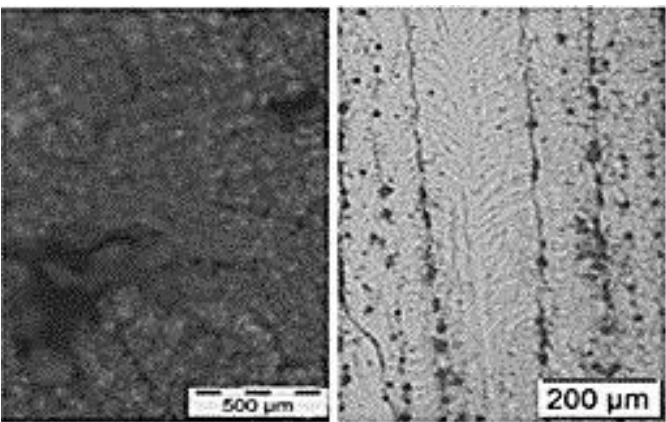

$P=5 \mathrm{~mm}$

$\dot{T}=2,81^{\circ} \mathrm{C} / \mathrm{s} ;$

$\mathrm{V}_{\mathrm{L}}=0,36 \mathrm{~mm} / \mathrm{s}$;

$\lambda_{1}=67,3 \mu \mathrm{m}$;

$\lambda_{2}=29,39 \mu \mathrm{m}$.
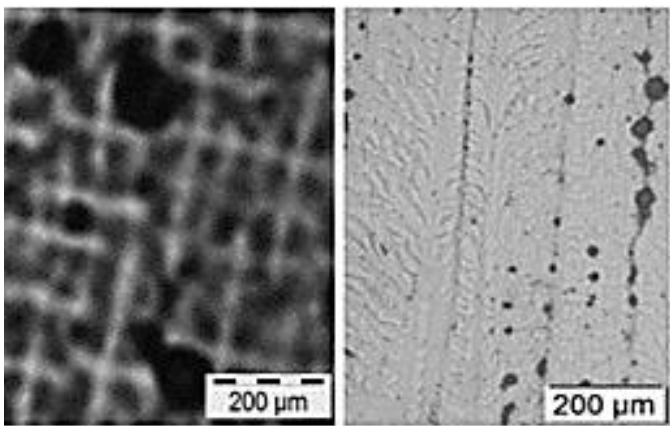

e)

$$
\begin{aligned}
& \mathrm{P}=70 \mathrm{~mm} ; \\
& \dot{T}=0,50^{\circ} \mathrm{C} / \mathrm{s} ; \\
& \mathrm{V}_{\mathrm{L}}=0,24 \mathrm{~mm} / \mathrm{s} ; \\
& \lambda_{1}=269,8 \mu \mathrm{m} ; \\
& \lambda_{2}=49,83 \mu \mathrm{m} .
\end{aligned}
$$

Figura 6: À esquerda tem-se as microestruturas transversais e, à direta, microestruturas longitudinais dos três superaquecemos da liga Al-3\%Mg investigadas: (a) e (b) $\Delta \mathrm{TV}=20^{\circ} \mathrm{C}$; (c) e (d) $\Delta \mathrm{TV}=40^{\circ} \mathrm{C}$; (e) e (f) $\Delta T V=60^{\circ} \mathrm{C}$.

Como é observado nas microestruturas os valores de $\lambda_{1}$ e $\lambda_{2}$ aumentaram com o aumento nos patamares de superaquecimento.

Na Figura 7 (a), é mostrado o gráfico da evolução de $\lambda_{1}$ (EDP) em função de $V_{L}$ para todos os superaquecimentos estudados e, na Figura 7 (b) apresenta $\lambda_{1}$ em função de $\dot{T}$ também para os mesmos superaquecimentos.
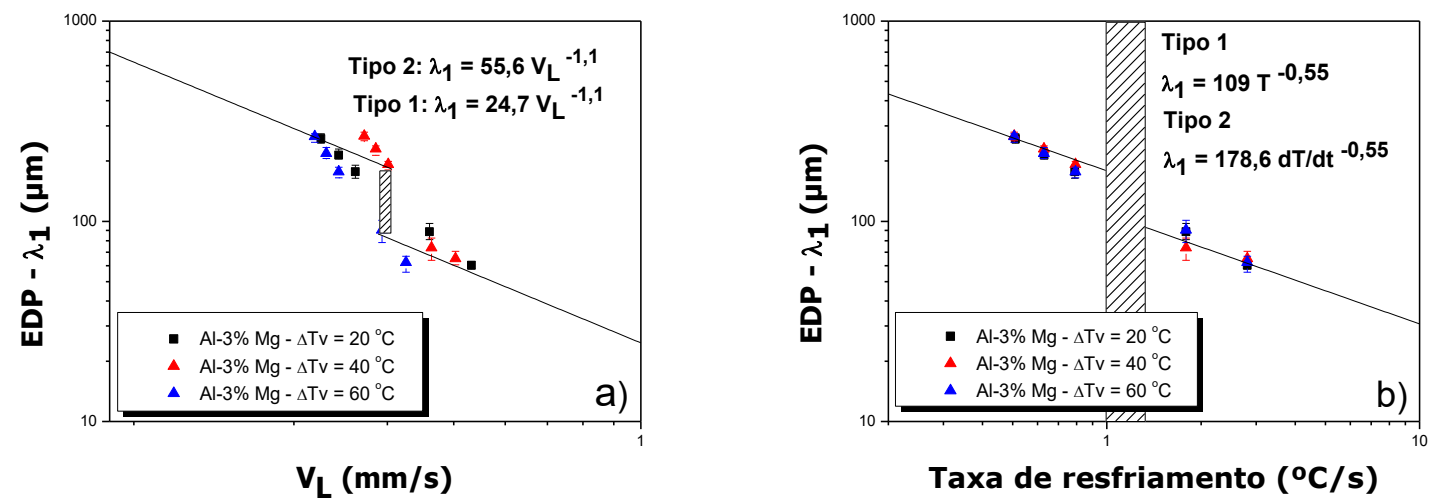

Figura 7: $\operatorname{EDS}\left(\lambda_{1}\right)$ para ambos superaquecimentos estudados em função de: a) $V_{L}$ e b) $\dot{T}$

Na Figura 8 (a) observa-se a evolução de $\lambda_{2}$ (EDS) em função de $V_{L}$ para os superaquecimentos estudados e na Figura 8 (b) também mostra $\lambda_{2}$ em função de $\dot{T}$ para os mesmos superaquecimentos. Pode-se afirmar que os superaquecimentos distintos afetaram na dinâmica de crescimento dos $\lambda_{1}$, pois as inclinações das curvas não são constantes. Em relação ao $\lambda_{2}$, os superaquecimentos não afetaram a dinâmica de crescimento pois as inclinações das curvas permaneceram constantes. 

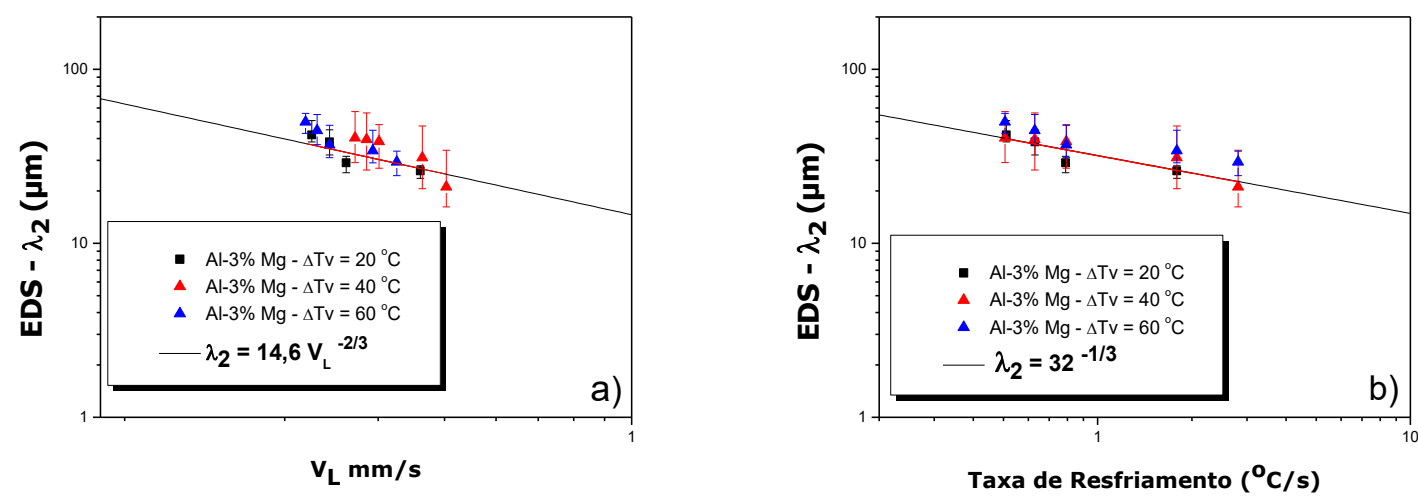

Figura 8: $\operatorname{EDP}\left(\lambda_{2}\right)$ para ambos superaquecimentos estudados em função de: a) $V_{L}$ e b) $\dot{T}$

\subsection{Caminho de Solidificação da liga Al-3Mg}

O caminho de solidificação em condições de equilíbrio da liga Al-3\%Mg é relativamente simples, e a microestrutura final de solidificação é constituída basicamente da fase $\alpha$-Al. A Figura 9 apresenta o caminho de solidificação calculado com o auxílio do software de termodinâmica computacional Thermo-Calc. Os valores para cada elemento foram obtidos a partir dos resultados de composição química provenientes dos ensaios de FRX, já apresentado. Para essa liga, observou-se que em condições fora do equilíbrio, as fases que estão presentes são, Al-a, Al-Mg- $\beta$ $\left(\mathrm{Al}_{3} \mathrm{Mg}_{2}\right)$ e $\mathrm{Mg}_{2} \mathrm{Si}$. Em relação a quantidade volumétrica dos intermetálicos ao longo do comprimento do lingote, pode-se afirmar que quanto maior for o espaçamento dendrítico maior será a quantidade volumétrica de intermetálicos nesses espaços. A fase $\mathrm{Mg}_{2} \mathrm{Si}$ possui uma elevada dureza de $650 \mathrm{HV}$, quase dez vezes superior à matriz a rica em Al. Essas partículas intermetálicas duras tem a habilidade em adotar uma morfologia curvada e revoluta em torno das ramificações dendríticas secundárias tornado 0 arranjo macroestrutural mais duro (Verna et al [11]).

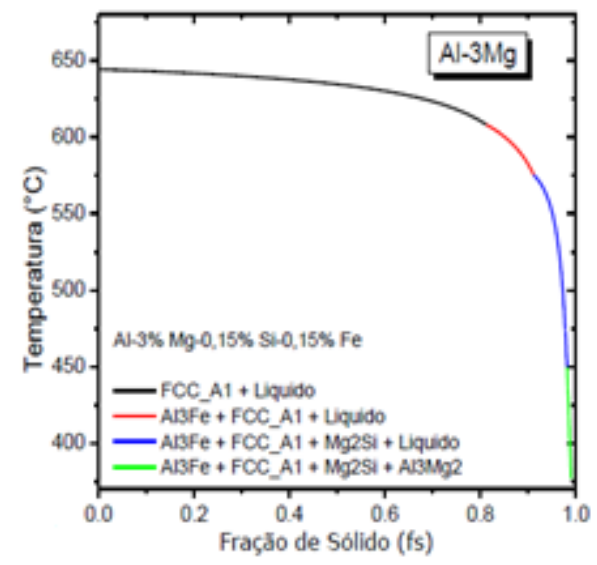

Figura 9: Caminho de solidificação, calculado a partir dos valores de composição obtidos nos ensaios de FRX para a liga $\mathrm{Al}-3 \% \mathrm{Mg}$

\subsection{Valores obtidos no Ensaio de microdureza Vickers (HV).}

A Figura 10 mostra os valores da microdureza $\mathrm{HV}_{0,5}$ da liga $\mathrm{Al}-3 \% \mathrm{Mg}$, para os três superaquecimentos estudados nas alturas de 5, 10,35, 50 e 70mm do comprimento dos lingotes em relação a base refrigerada. Observa-se que, para valores crescentes da posição, as microdurezas aumentaram. São observados maiores valores da microdureza HV para menores $\Delta \mathrm{T} v$, pois, com um maior superaquecimento a liga solidifica-se numa condição mais próxima do equilíbrio favorecendo a segregação e o 
aumento dos braços dendríticos. A macrossegegação e defeitos como o rechupe são observados somente nas ultimas seções de solidificação, fora da região de estudo, não interferido assim na estrutura final analisada.

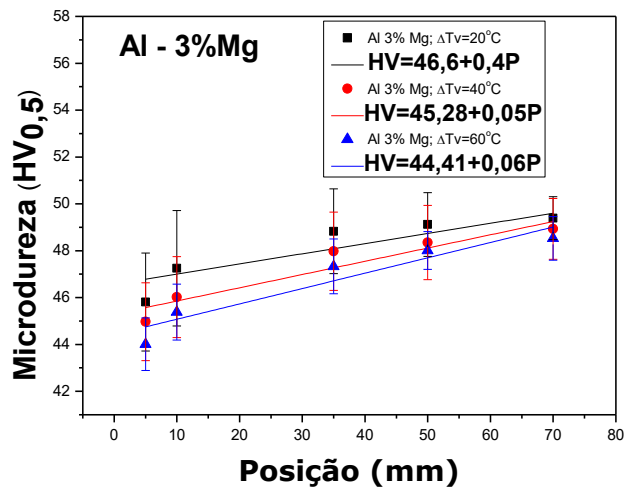

Figura 10: Perfil da microdureza $\mathrm{HV}_{0,5}$ em função da posição nos lingotes na liga Al-3\%Mg: a) $\Delta \mathrm{T}_{\mathrm{V}}=$ $20^{\circ} \mathrm{C}$; b) $\Delta \mathrm{T} v_{\mathrm{v}}=40^{\circ} \mathrm{C}$; c) $\Delta \mathrm{Tv}=60^{\circ} \mathrm{C}$.

Os perfis de microdureza em função de $\lambda_{2}$, para os três superaquecimentos estão apresentados na Figura 11. A partir dos perfis de microdurezas, pode-se afirmar que o sutil aumento da microdureza, com o aumento de $\lambda_{2}$, deveu-se mais aos intermetálicos que atuarão como barreiras ao movimento das discordâncias, que provocam a deformação plástica. A fase $\mathrm{Mg}_{2} \mathrm{Si}$, possui baixa densidade $\left(1,98 \mathrm{~g} / \mathrm{cm}^{3}\right)$, e um excelente reforço macroestrutural para as ligas-leves à base de Alumínio (Bray [12]). Um outro fator chave associado ao aumento da dureza dessas ligas é a solução sólida de Mg na matriz de Al. A diminuição em Mg sob solução sólida contribui para a diminuição observada na microdureza.

A Figura 11 apresenta também a equação tipo Hall-Petch $\left(H V=H_{0}+K \cdot \lambda_{2}{ }^{-1 / 2}\right)$ que pode ser empregada para correlacionar a microdureza Vickers $(\mathrm{HV})$ como função do espaçamento dendrítico secundário, ou seja, microdureza versus o inverso da raiz quadrada de $\lambda_{2}$.

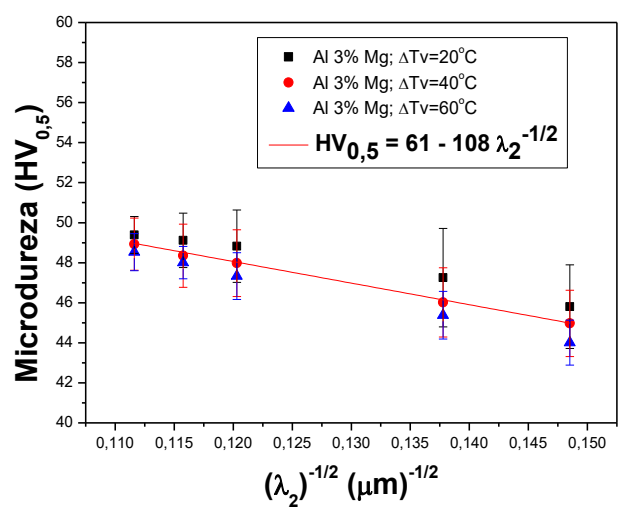

Figura 11: Microdureza $\mathrm{HV}_{0,5}$ em função espaçamentos dendríticos secundários (EDS ou $\lambda_{2}$ ) da liga Al-3\%Mg: a) $\Delta \mathrm{TV}_{\mathrm{V}}=20^{\circ} \mathrm{C}$; b) $\Delta \mathrm{T}_{\mathrm{V}}=40^{\circ} \mathrm{C}$; c) $\Delta \mathrm{T}_{\mathrm{V}}=60^{\circ} \mathrm{C}$.

\section{CONCLUSÃo}

A partir do trabalho pode-se concluir que:

A variação superaquecimento $(\Delta \mathrm{TV})$ não exerceu influência direta nas variáveis térmicas de solidificação de forma que à medida que se elevou o $\Delta T \mathrm{v}$, a taxa de resfriamento praticamente não se alterou no ponto onde ocorre a Transição das Estrutura Colunar - Equiaxial (TCE), mostrando que para a liga $\mathrm{Al}-3 \% \mathrm{Mg}$, o superaquecimento não teve influência na posição da TCE. 
Quanto à microestrutura, foram propostas leis experimentais de crescimento para os três superaquecimentos estudados da liga Al-3\%Mg, relacionando $\lambda_{1}$ e $\lambda_{2} \mathrm{em}$ função de $V_{L}$ e de $\dot{T}$. Pode-se observar que maiores são os espaçamentos interdendríticos para maiores superaquecimentos. $E$ foram também propostas leis de crescimento para $\lambda_{1}$ e $\lambda_{2}$ em função de $V_{L}$ e de $\dot{T}$.

A microdureza Vickers apresentou uma elevação em função do aumento das posições e do $\lambda_{2}$, para os três superaquecimentos da liga Al-3\%Mg estudados dos lingotes e também com a diminuição do $\Delta \mathrm{T} v$.

\section{REFERÊNCIAS}

[1]HUNT, J.D.; LU, S.Z.: Numerical modeling of cellular array growth: spacing and structure predictions, Metallurgical Materials Transactions, v. 27A, n. 3, (1996), 611623.

[2]CRUZ, K.A.S., CHEUNG, N., GARCIA, A., "Parâmetros térmicos, macroestrutura e microestrutura na solidificação direcional da liga Al-20\%Sn", Tecnologia em Metalurgia e Materiais, v. 4, n. 4, pp. 21-26, Apr. 2008

[3]REIS, B.P., FRANÇA, R.P., SPIM, J.A., et al, "The effects of dendritic arm spacing (as-cast) and aging time (solution heat-treated) of Al-Cu alloy on hardness", Journal of Alloys and Compounds, v. 549, pp. 324-335, Feb. 2013.

[4]BOUCHARD, D., KIRKALDY, J.S., "Prediction of dendrite arm spacings in unsteadyand steady-state heat flow of unidirectionally solidified binary alloys", Metallurgical and Materials Transactions B, v. 28B, pp. 651-663, Aug. 1997

[5]GAFUR, M.A.; NASRUL HAQUE, M.; NARAYAN PRABHU, K.: Effect of chill thickness and superheat on casting/chill interfacial heat transfer during solidification of commercially pure aluminium, Journal of Materials Processing Technology, v. 133, n. 3, (2003), 257-265.

[6]CHEUNG, N.; FERREIRA, I. L.; PARIONA, M.M.; QUARESMA, J. M. V.; GARCIA, A.: Melt characteristics and solidification growth direction with respect to Gravity affecting the interfacial heat transfer coefficient of chill castings, Materials and Design, v. 30, (2009), 3592-3601.

[7]SIQUEIRA FILHO, C. A., Influência de Parâmetros Térmicos de Solidificação na Transição Colunar/Equiaxial [Tese de Doutorado]. Campinas: Universidade de Campinas; 2002.

[8]SPINELLI, J.E., ROSA, D.M., FERREIRA, I.L., et al, "Influence of melt convection on dendritic spacings of downward unsteady-state directionally solidified Al-Cu alloys", Materials Science and Engineering A, v. 383, pp. 271-282, Oct. 2004

[9]DANTAS, P.P., Caracterização de Ligas Hipoeutéticas de Al-Ni Solidificadas Unidirecionalmente [Dissertação]. Brasília: Universidade Federal de Brasília, 2014. [10]GÜNDÜZ, M.; ÇARDILI, E.; Directional Solidification of Aluminium-Copper Alloys, Materials Science and Engineering A. v. 327, p. 167-185, 2002.

[11]VERMA, A., KUMAR, S., GRANT, P.S., e O'REILLY, K. A. Q. Influence of cooling rate on the Fe intermetallic formation in an AA6063 Al alloy. Journal of Alloys and Compounds, v. 555, p. 274-282, 2013.

[12]BRAY, J. W. Aluminum Mill and Engineered Wrought Products. In Handbook, Metals. "vol. 2." Properties and Selection: Nonferrous Alloys and Special-Purpose Materials. v. 713. p. 124148. 1992. 